\title{
تاثير اسلويي التلمذة المعرفية وخطة كيلر في تعلم مهارة الارسال من الاسفل المواجة الامامي في الكرة الطائرة لاى طلاب الصف الاول متوسط

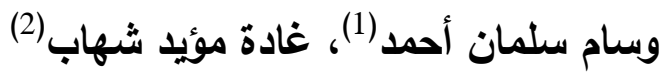 \\ تأريخ تقيم البحث: (2020/2/11)، تأريخ قبول النثر (2020/3/12).
}

\begin{abstract}
المستخلص
أن أسلوبي التلمذة المعرفية وتفريد التعليم من الاساليب التعليمية التي قد تكون مناسبة والتي لها تأثير ايجابي في تعليم مهارات الكرة الطائرة، اذ ان لكل اسلوب خصوصيته في التعلم وقد هدف البحث في التعرف على تأثثر اسلوبي التلمذة المعرفية وخطة كيلر في تعلم مهارة الارسال من الاسفل المواجة الامامي في الكرة الطائرة لدى طلاب الصف الاول المتوسط، وقد افترض الباحثان ان هناك فروق ذات دلالة احصائية بين الاختبارات القبلية والبعدية لمجاميع البحث الثلاثة في تعلم مهارة الارسال من الاسفل في الكرة الطائرة، وان هناك فروق ذات دلالة احصائية في الاختبارات البعدية لاى عينة البحث، نم استخدام المنهج التجريبي باسلوب المجموعات الثلاث (التجريبيتان والضابطة) وتمنلت العينة بطلاب الصف الاول في متوسطة محمد باقر الصدر للبنين/ بغداد/ الكرخ الثالثة، البالغ عددهم(112) طالب، استخدم اسلوب التلمذة المعرفية مع المجموعة التجريبية الاولى واسلوب خطة كيلر مع المجموعة التجريبية الثانية والاسلوب الامري مع المجموعة الضابطة، وبعد جمع البيانات ومعالجتها احصائياً تم التوصل الى مجموعة استتاجات اهمها ان اسلوبي التلمذة المعرفية وخطة كيلر (تفريد التعليم) لهما تاثير فعال في تعلم مهارة الارسال من الاسفل مقارنة بالاسلوب المنبع في الدرس. الكلمات المفتاحية: تفريد التعليم، النظرية البنائية الاجتماعية
\end{abstract}

\section{ABSTRACT \\ The Effect of Cognitive Apprenticeship Style and Keller Plan on Learning Volleyball Underarm Serving In First Year secondary School Students}

The research aim at identifying the effect of cognitive apprenticeship and keller plan on learning underarm serving in volleyball for $1^{\text {st }}$ grade secondary school students. The researchers hypothesized statistical differences between pre and posttests in all three groups in learning volleyball serving as well as statistical differences in posttests. The researchers used the experimental method on three groups; two experimental and one controlling group on (112) first grade secondary school students (Mohammed Baqer Al Sedar). The cognitive apprenticeship was used on the first experimental group, Keller plan on the second group and the command style on the controlling group. The data was collected and treated using proper statistical operations to conclude that cognitive apprenticeship and keller plan have a great effect on learning serving in volleyball compared to the style followed by the teacher.

Keywords: individualized learning, social building theory.

(1) طالب دراسات عليا (الدكتوراه)، جامعة بغداد، كلبة التربية البدنية وعلوم الرياضة. (wisamdimond@gmail.com) Wissam Salman Ahmed, Post Graduate Student (P.HD), University of Baghdad, College of Physical Education and Sport Sciences, (wisamdimond@gmail.com).(+96407729482082)

(2) أستاذ، دكتوراه تربية رياضية، جامعة بغداد، كلبة التربية البدنية وعلوم الرياضة. (Dr.ghada@cope.uobaghdad.edu.iq) Ghada Moayad Shihab, Prof (PH.D), University of Baghdad, College of Physical Education and Sport Sciences, (Dr.ghada@cope.uobaghdad.edu.iq) (+96407713311764). 
ان اعداد المتعلم وتدريبه على حل الصعوبات التي تواجهه في عملية التعليم يتطلب من المعلم ان يختار الطريقة او الاسلوب التي تتتاسب وطريقة تقكيره وتكثف عن طاقاته الكامنة من خلال تقديم انشطة تربوية تقدم اليه داخل الوحدة التعليمية متدرجة في الصعوبة تجعله قادر في الاعتماد على نفسة وتحمل المسؤولية والتقة في حل المشكلات وتتمية تفكيره.

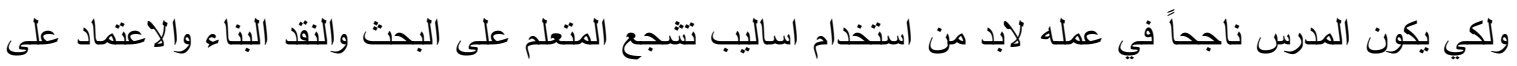

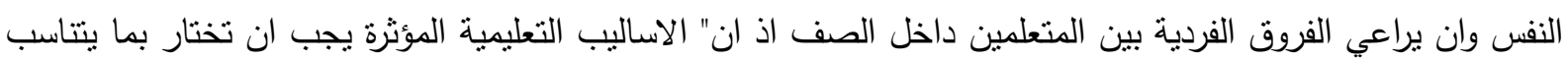
مع حاجات وخصائص المتعلمين والمنهج والاهداف المرغوب التوصل اليها بما يتفق مع الامكانات المادية والبشرية

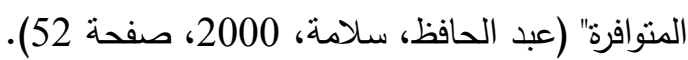
ويرى الباحثان ان اسلوبي التلمذة المعرفية وتفريد التعليم من الاساليب التعليمية التي قد تكون مناسبة والتي لها تأثير

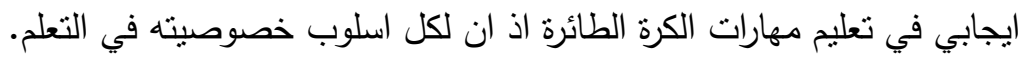

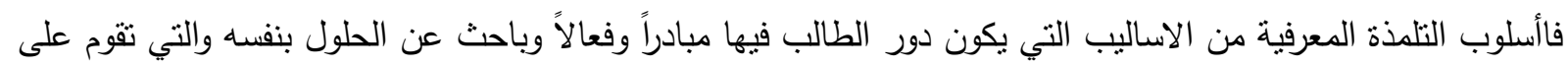
اساس عمل الطالب في مجموعات وفرق وان يكتسب المهارات المعرفية من خلال المشاركة التعاونية من خلال سته مراحل تعليمية وهي( النمذجة- التصقيل- التأمل- تطبيق المهارة- التعبير - الاستكثاف). (محمد، حمدي البيطار، 2014،

صفحة 185).

اما اسلوب تفريد التعليم (خطة كيلر) فهو من الاساليب المهمة التي نراعي الفروق الفردية بين المتعلمين ويعطي الفرصة للمتعلم ان يتعلم وفق قدراته الذاتية، اي يكون التعليم ذاتي مستقل ويستتد الى مواد تعليمية معدة وكاملة لتيسير

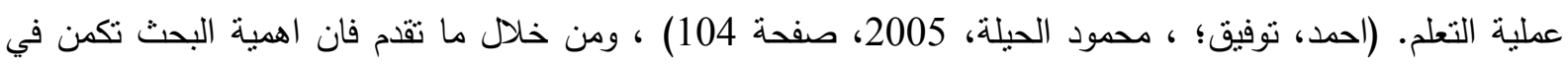
اختبار هذين الاسلوبين وهما اسلوب التلمذة المعرفية الذي يعد احد اسالبب النظرية البنائية الاجتماعية التي تؤكد على مبلى مبدأ

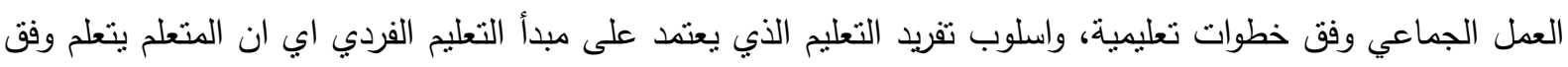
سرعته الذاتية، باعتبارها من الاساليب التي تعتمد على اثراك الطلاب بشكل فاعل ونشط يتتاسب مع قدراتهم ورغباتهم وتدعم عملية التعلم. وفي هذا المجال اجريت دراسات متعدده منها دراسة (صباح، عائد؛ واخرون، 2008) توصلت الى أن (أنموذج

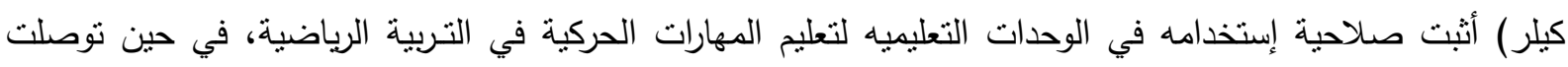

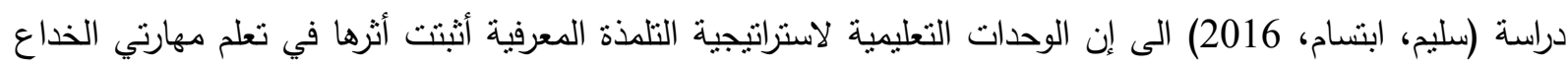
البسيط والتصويب من السقوط الأمامي في كرة اليد، وقد تثابهت الدراسة الحالية مع الدراسات السابقة في اختبار المتغير المستقل المتمنل باسلوب التلمذة المعرفية وخطة كيلر واختلفت معها في المتغير التابع المتمثل بالعينة والمهارات

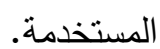

ويرى الباحثان ان اغلب المدارس تعتمد في عملية تعليم المهارات في الالعاب الرياضية بشكل عام ولعبة الكرة الطائرة بشكل خاص على اساليب التعلم التي تعتمد على عملية تلقين المعلومات وحفظها واسترجاعها وتذكرها اكثر من اهتمامها

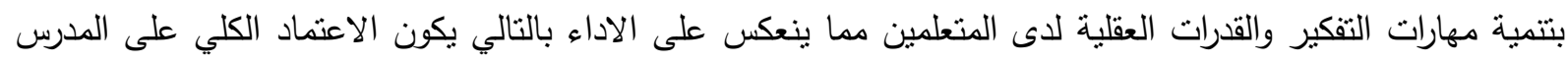

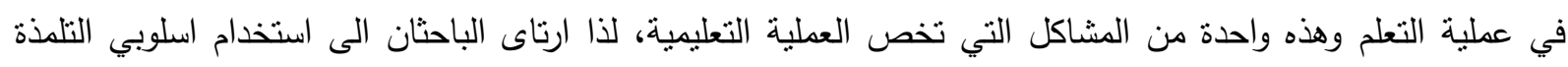

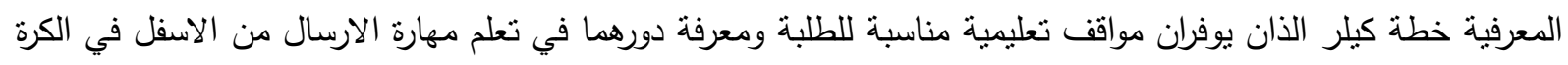
الطائرة.

وهدف البحث في التعرف الى تأثثر اسلوبي التلمذة المعرفية وخطة كيلر في تعلم مهارة الارسال من الاسفل المواجة الامامي في الكرة الطائرة لاى طلاب الصف الاول المتوسط وقد افترض الباحثان ان هناك فروف ذات دلالة احصائية بين 
الاختبارات القبلية والبعدية لمجاميع البحث الثلاثة (التجريبيتان والضابطة) في تعلم مهارة الارسال من الاسفل المواجة

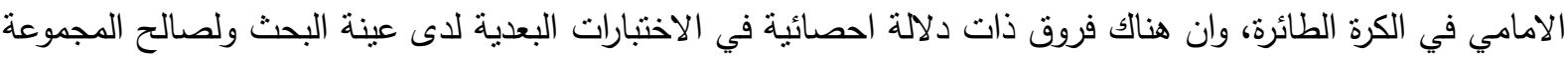
التجريبية الاولى، ان موضوع التلمذة المعرفية وخطة كيلر في بعض الالعاب الرياضية كان بحمل اهية كبيرة لدى الكثير من الباحثين في الدراسة.

الطريقة والادوات:

أُستخدم المنهج التجريبي بتصميم المجموعات الثلاثة (مجموعتان تجريبيتان ومجموعة ضابطة) ذات الاختبار القبلي والبعدي وذلك لملائمته مشكلة البحث، وتم اختيار مجتمع البحث بالطريقة العمدية والمتمثل بطلاب الصف الاول في

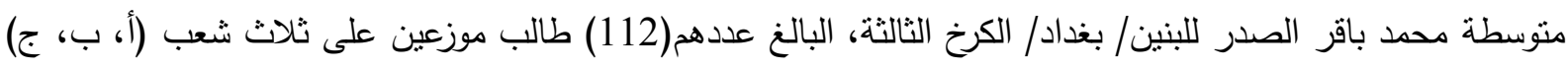
وعن طريق القرعة تم اختيار (15) طالب من كل شعبة ليمنل عينة البحث اذ بلغ عددها(45) طالب بنسبة ( 17،40\%) من مجتمع البحث وبعد تقسيم العينة الى ثلاث مجاميع نم اجراء الاختبار القبلي لمهارة الارسال من الاسفل المواجة عادية

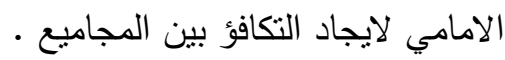
وتم اجراء ثلاث تجارب استطلاعية على عينة من طلاب الصف الاول متوسط البالغ عددهم(5) طلاب خارج عينة البحث، تجربة استطلاعية اولى لأسلوب التلمذة المعرفية وتجربة استطلاعية ثانيه لأسلوب تفريد التعليم (خطه كيلر) بتاريخ

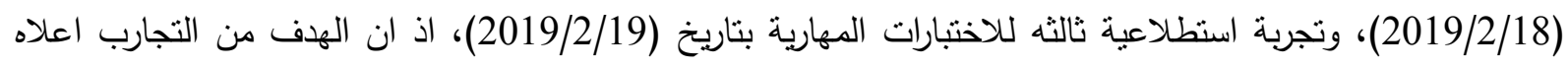
هو لمعرفة الوقت المستغرق لتتفيذ الاختبارات المهارية والمكان المناسب لتصوير العينة لغرض تقويم الاداء. وبعدها اجري الاختبار القبلي لعينة البحث بعد اعطاء وحدة تعريفية عن المهارات لكل مجموعة زمنها (30 د) لاخذ فكرة عن المهارات، تم تطبيق التجربة الرئيسة في الفصل الدراسي الثاني لعام(2019م) واستغرق تتفيذ الوحدات التعليمية لمدة (ثهرين واسبوع) ابتداءا من ناريخ (2019/2/24) لغاية (2019/5/5)، بواقع (2) وحدة تعليمية وحدتان تعليميتان في لنياني

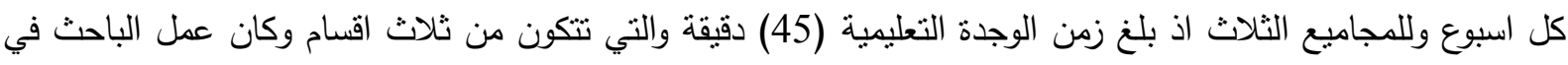
القسم الرئيسي من الوحدة التعليمية وزمنها (30) دقيقه، ونم تعليم المهارات الحركية للمجموعة التجريبية الاولى وفق أسلوب وند

$$
\text { التلمذة المعرفية وعلى النحو الاتي: }
$$

النمذجة: في هذه المرحلة قام مدرس المادة بشرح المهارة وعرضها مع التركيز على تفاصيل المهارة بشكل دقيق.

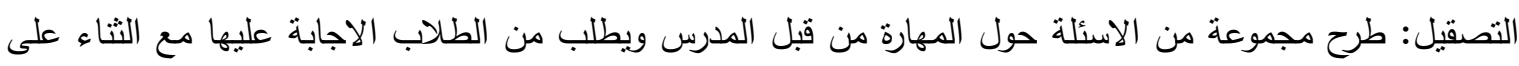

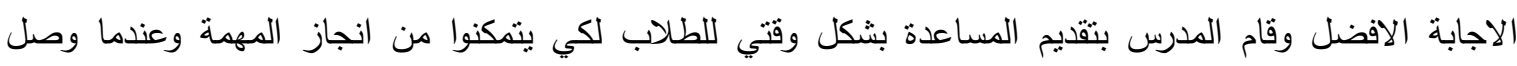
المتعلم الى مقدرة كافية للإجابة على الاسئلة بشكل صحيح توقفت المساعدة وبدأ بزيادة صعوبة الاسئلة اما دور المدرس فهو التجوال بين المجاميع. تطبيق المهارة: تم اداء المهارة من قبل الطلاب اما دور المدرس فهو مراقبة اداء الطلاب في تتفيذ المهام التعليمية وتقييم اداءهم وتتجيع المجموعة الافضل والمجموعة التي اظهرت اداءاً متميزاً نم اعطاءها تغذية راجعة نعزيزية والتي تظهر اداءاً متدنياً نم اعطاءها تغذية راجعة تصحيحية. التأمل: في هذة المرحلة قام المدرس باعادة عرض الاداء امام الطلاب مرة ثانية وقام كل طالب بمقارنة اداءه بأداء

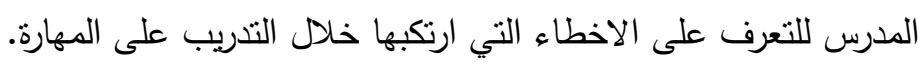

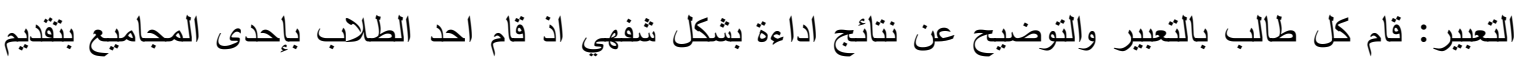
سؤال عن المهارة وتم الاجابة عليه من قبل المجاميع مع تصحيح الخطاء والثناء على الاجابة الصحيحة. الاستكثاف: نم التوصل الى الاداء الصحيح وذلك من خلا عمل عن اختبار تحريري عن طريق مجموعة من الاسئلة تطرح للطالب حول المهارة وتم الاجابة عليها، وتقييم جميع الآراء التي طرحت في المراحل السابقة من قبل المدرس. 
وهناك تتابع وتسلسل للمحتوى التعليمي لاسلوب التلمذة المعرفية وحسب مبدئين: مبدأ الزيادة في التعقيد/ ويقصد به التسلسل من البسيط الى المركب للمهام التعليمية. مبدأ الزيادة في التتوع / ويقصد به التنوع في مختلف المهارات والاساليب التي تقدم للطالب كي يتعلموا التمييز بين

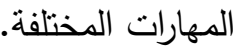
اما المجموعة التجريبية الثانية التي تتعلم المهارات الاساسية بأسلوب تفريد التعلم (خطة كيلر) فكانت على النحو الاتي: - n قام الباحث بأعداد دليل الطالب الهدف منة ارشاد المتعلم خلال الوحدة التعليمية والذي يحتوي على المهارات الاساسية وهي مهارة الارسال من الاسفل المواجة الامامي في الكرة الطائرة، موضحا فيها اقسام المهارة والاخطاء الثائعة والتمارين المستخدمة واسئلة التقويم الذاتي التي تتعلق بكل مهارة. قام كل طالب بتعلم المهارة حسب قابليته الذاتية باعتماده على الداليل المطبوع. في نهاية كل وحدة تعليمية هناك اختبارات توفر تغذية راجعة للمتعلم اذ اعتمد المدرس على معيار محكي بنسبة 80\% تم الاعتماد عليه لغرض الانتقال الى مهارة اخرى، وهذا تم عن طريق اختبارات قصيرة اختبار مهاري واختبار معرفي ورقة وقلم يتضمن اسئلة معدة مسبقاً موجودة في الدليل. اما المجموعة الضابطة فتم تعليمها المهارات وفق اسلوب المدرس وهو الاسلوب الامري، وبعد الانتهاء من تتفيذ الوحدات التعليمية اجريت الاختبارات البعدية في /5/6 2019 لمجاميع البحث الثنلاث في مهارة الارسال من الاسفل مهن

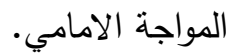
وتم استخدام اختبار مهارة الإرسال المواجه من الأسفل: (محمود، نسيمة والي، 2006، صفحة 308)

الجدول(1) يبين نتائج الاختبارات القبلية والبعدية لمجاميع البحث لمهارة الارسال من الاسفل المواجة الامامي

\begin{tabular}{|c|c|c|c|c|c|c|c|c|}
\hline \multirow{2}{*}{ الدلالة } & \multirow{2}{*}{ 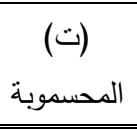 } & \multirow{2}{*}{ ف هـ } & \multirow{2}{*}{ ف } & \multicolumn{2}{|c|}{ الاختبار البعدي } & \multicolumn{2}{|c|}{ الاختبار القبلي } & \multirow{2}{*}{ المتغيرات } \\
\hline & & & & $\varepsilon$ & w & $\varepsilon$ & س & \\
\hline 0.000 & 29.787 & 0.174 & 5.200 & 0.516 & 7.533 & 0.487 & 2.333 & المجموعة التجريبية 1 \\
\hline 0.000 & 39.287 & 0.106 & 4.200 & 0.507 & 6.600 & 0.507 & 2.400 & المجموعة التجريبية2 \\
\hline 0.000 & 16.877 & 0.181 & 3.066 & 0.593 & 5.266 & 0.414 & 2.200 & المجموعة الضابطة \\
\hline
\end{tabular}

معنوي > (0.05) عند درجة حرية (14).

الجدول(2) يبين نتائج تحليل التباين الاحادي بين مجاميع البحث واختبار (L.S.D) بين مجاميع البحث في

\begin{tabular}{|c|c|c|c|c|c|c|}
\hline \multicolumn{7}{|c|}{ الاختبار البعدي } \\
\hline مستوى الخطأ & الخطاء المعياري & فرق الاوساط & المجموعة & مستوى & قليمة (ف) & المهارات \\
\hline 0.000 & 0.197 & $0.933^{*}$ & الاولى - الثانية & \multirow{3}{*}{0.000} & \multirow{3}{*}{66.620} & \multirow{3}{*}{ الآسفل من } \\
\hline 0.000 & 0.197 & $2.266^{*}$ & الاولى - الضابطة & & & \\
\hline 0.000 & 0.197 & $1.333^{*}$ & الثانية- الضابطة & & & \\
\hline
\end{tabular}

معنوي عند> (0.05) عند درجة حرية(42،3). 
اظهرت نتائج تحليل البيانات الاحصائية في الجدول (1) لمعرفة معنوية الفروق بين الاختبارين القبلي والبعدي وجود فروق دالة احصائياً في تعلم المهارات قيد البحث ولصالح الاختبار البعدي للمجاميع الثناثة التجريبيتان والضابطة ويعزو الباحث ذلك الى المنهج التعليمي وما يحتويه من تتوع في الاساليب التعليمية واتباع الخطوات الصحيحة وتطبيق مبادى التهاء

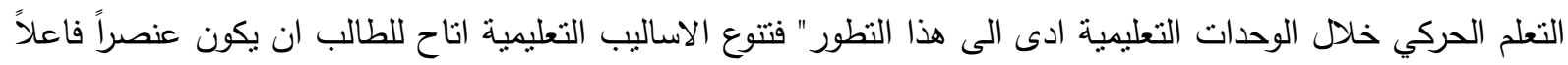

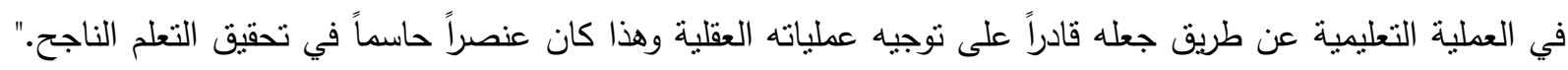
(محمود، دعاء، 2016، صفحة 83). اذ ان المجموعة التجريبية الاولى باسلوب التلمذة المعرفية ساعد الطلاب على تحمل المسؤولية من خلال انخراطهم في الاثشطة التي وفرتها مراحل التلمذة المعرفية التي ساعدتهم على التفاعل النشط في الدرس وان تطبيق التمرينات

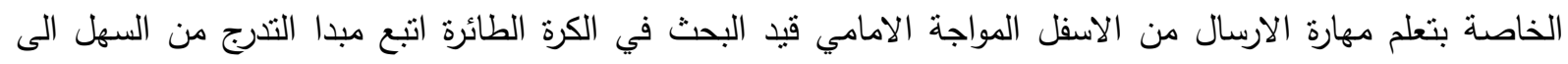

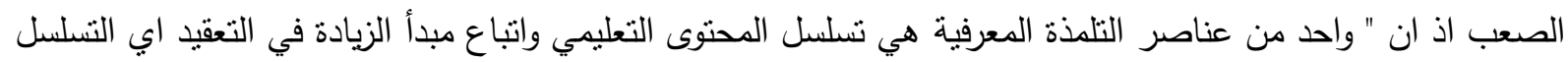

من البسيط الى المركب للمهام التعليمي (Sbehrman, brown.j., 1997, p. 34) كما ان المجموعة التجريبية الثانية التي تعلمت وفق (خطة كيلر) حققت نتائج ايجابية كون ان الطالب يختار المستوى تبعاً لقدراته واستعداداته اذ ان هناك فروق فردية بين الطلاب داخل الصف الواحد فقد اعطى اسلوب تفريد التعليم فرصة إنها لكل طالب لتحقيق الهدف في الزمان والمسنوى الذي يناسبه اذ ان" مراعاة الفروق الفردية داخل الجماعة والتفريق بين

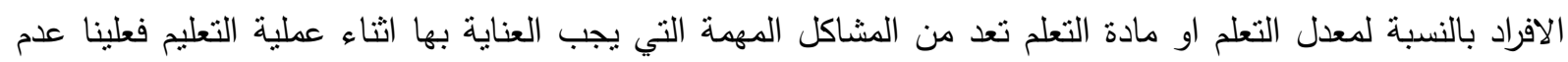
اخضاع الطلبة لنفس المستوى من التحصيل او الانجاز بل استخدام اجراءات تتضدن نوفير انشطة تعليمية منتوعة لتعلم

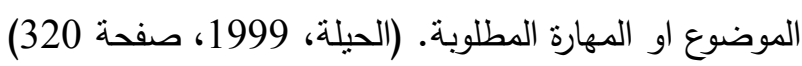
وفي ضوء النتائج الخاصة بتحليل التباين الموضحة في الجدول(2) ونتائج اقل فرق معنوي( L.S.D) الموضحة في الجدول(3) وجود فروق دالة احصائياً بين المجاميع الثلاث في تعلم المهارات قيد البحث ولصالح المجموعة التجريبية الاولى (التلمذة المعرفية) تلته المجموعة التجريبية الثانية (خطة كيلر) ثم المجموعة الضابطة، ويعزو الباحث ذللك ان فئل اسلوب التلمذة المعرفية ساعد الطلاب في التغلب على المشكلات واكتساب المعرفة وجعله اكثر نشاطاً من خلال تتشيط

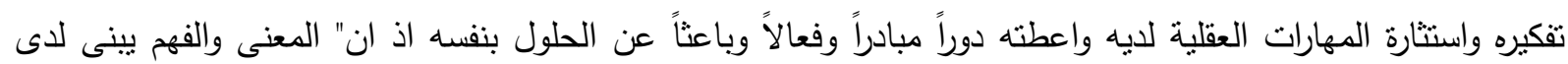

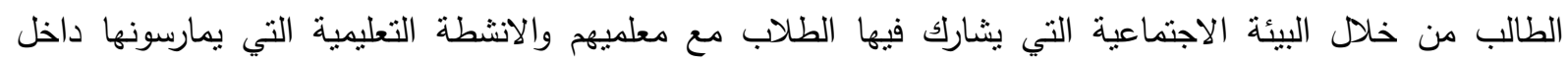
الفصول الدراسية" (سليمان، زيد؛ عيسى، احمد، 2016، صفحة 64). كما ان" التقويم المستمر والمراقبة الدورية المنتظمة للمعلم والمدرس الجيد تعتبر من الحوافز المهمة للنمو الاختصاصي والمحافظة على الكفاءة فقط بل مساعدتهم على

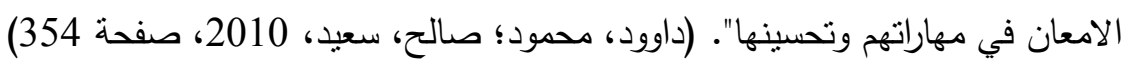
فالتلمذة المعرفية تقوم على أسس مهمة ومنها عمل الطلاب بتتكيل فرق لمناقثة وحل المشاكل وانتراك المدرب معهم من خلال تقديم الدعم لهم لتحقيق التكامل والنجاح في العمل. كما يعزو الباحث سبب تطور المجموعة التجريبية الاولى الى الخطوات التي اتبعها الطلاب في اسلوب التلمذة المعرفية اذ اعطت فرصة لهم في المناقثة والحوار مع الزملاء في نفس المجموعة من خلال العمل في مجموعات وتتمية

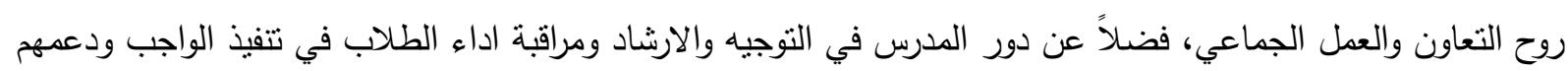
عند الحاجة لزيادة مستوى الفهم فدور المدرس كان ايجابياً وميسر لعملية التعلم وليس فقط ملقن للمعلومات " فالتعلم عملية

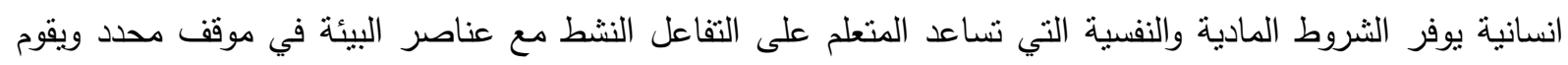

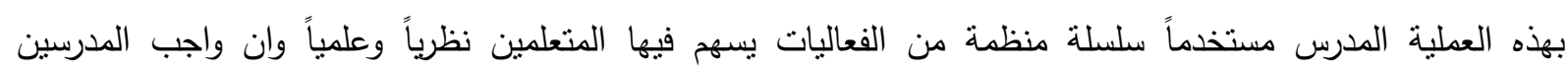
لاتتحصر في غرفة الصف وانما تعدت الى خارجها وصار المدرس لايستطيع النجاح في عمله مالم ينبين له طبيعة طلابه 
وخصائصهم وان يكون قادر على ادارة الصف ومراعاة الفروق الفردية بينهم ونتجيعهم ومعرفة قدراتهم واحتياجاتهم والععل

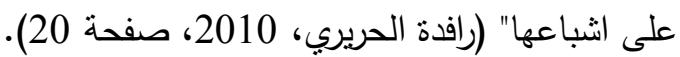

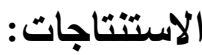

اظهرت النتائج في الاختبارات البعدية نطوراً واضحاً لمجاميع البحث الثلاث (التجريبينان والضابطة) في تعلم مهارة الارسال من الاسفل المواجة الامامي مقارنةً مع الاختبارات القبلية.

تفوق المجموعة التجريبية الاولى (النلمذة المعرفية)على المجموعة التجريبية الثانية (خطة كيلر) وكذلك المجموعة الثالثة في تعلم مهارة الارسال من الاسفل المواجة الامامي في الكرة الطائرة.

المصادر

رافدة الحريري. (2010). طرق التدريس بين التقليد والتجديد (المجلد 1). عمان: دار الفكر للنشر والتوزيع.

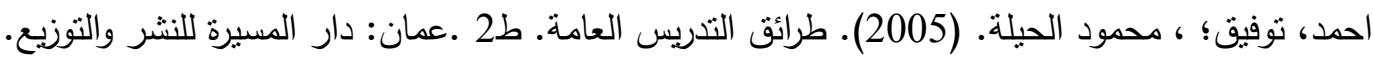
الحيلة. (1999). التصميم التعليمي نظرية وممارسة. عمان: دار المسيرة للنشر والتوزيع والطباعة. داوود، محمود؛ صالح، سعيد. (2010). الاتجاهات الحديثة في التربية الرياضية. اربيل: مطبعة المنارة.

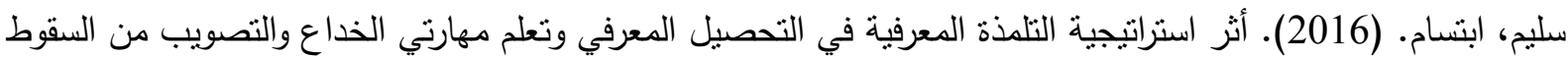
بكرة اليد للطالبات. بغداد: كلية التربية البدنية وعلوم الرياضة للبنات.

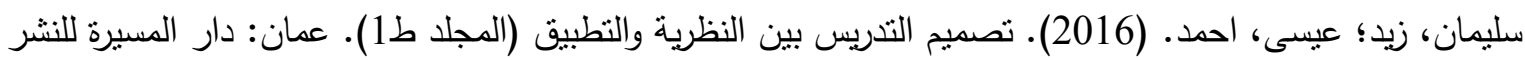
والتوزيع.

صباح، عائد؛ واخرون. (2008). تاثير انموذج كيلر في تعلم مهارتي الاستقبال والارسال في الكرة الطائرة. مجلة الفتح. عبد الحافظ، سلامة. (2000). الوسائل التعليمية والمنهج. عمان: دار الفودئ الفكر للنشر والتوزيع.

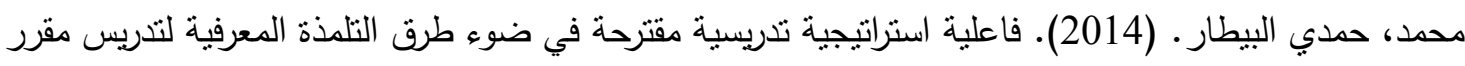
تكنولوجيا المياه والصرف الصحي في تتمية التحصيل الدراسي ومهارات التفكير الناقد لدى طالب الصف الاول الثانوي الصناعي،( مجلة كلية التربية بالسويس. كلية التربوية .

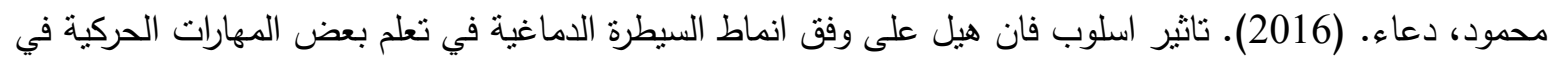
الجمناستلك الفني للنساء. جامعة بغداد: كلية التربية البدنية وعلوم الرياضئة. محمود، نسيمة والي. (2006). الاكتثاف الموجه وتدريس مهارات الكرة الطائرة وأثرها على التحصيل المهاري. الاسكندرية: دار الوفاء لانيا الطباعة والنشر •

Sbehrman, brown.j. (1997). Effectiveness of Cognitive Apprenticeship Instructional

Methodsin College Automotive Technology Classrooms. Industri Teacher Education, 34. 